\title{
Potential Use of Chemically Synthesized Iron-Pyrite Nanoparticles in Enhancing Plant Growth ${ }^{\dagger}$
}

\author{
Shrimathi V. ${ }^{1}$, Ranjith Kumar D.M. ${ }^{1}$, Ramesh Kumar V. ${ }^{1}$, Reji J.V. ${ }^{2, *}$ \\ 1 Department of Biotechnology, Sathyabama University, Chennai \\ 2 Department of Botany, Madras Christian College, Tambaram, Chennai \\ * Correspondence: jvreji@gmail.com; \\ $\dagger$ Presented at International e-Conference on Bioengineering for Health and Environment (ICBHE 2020)
}

Received: 5.07.2020; Revised: 10.07.2020; Accepted: 12.07.2020; Published: 15.07.2020

\begin{abstract}
Trouncing of seed dormancy and retarded plant growth is a major accomplishment in the sustainable production of agricultural crops. Generally, the activation of the Brassinosteroid (BR) pathway leads to an increase in the breaking of dormancy during seed development and germination. Pyrite in the water system contains trace amounts of ferrous, ferric, sulfate, and hydrogen peroxide ions, thereby forming an acidic milieu, which activates the Brassinosteroid (BR) pathway by the signaling action of hydrogen peroxide ions. Chemical synthesis of iron-pyrite nanoparticles, its characterization followed by testing of its efficacy in activation of BR pathway upon seed treatments, its validation through phytochemical analysis, and GC-MS studies are the main focus of this research work. The nanoparticle in the water system was also studied for its effect on Amylase activity in seed upon nanoparticle treatment. An increase in the amylase activity ultimately resulted in enhanced seed germination by breaking dormancy at a faster rate. The metabolic efficiency and possible toxicity of the plants generated from seed treated with nanoparticles were assessed through bio-chemical methods to corroborate the enhanced growth in plants and the harmlessness of the plants for consumption. The entire study was carried out with seeds of Raphanus sativus, as they have the ability to acclimatize in different agro-climatic conditions.
\end{abstract}

Keywords: seed dormancy; iron-pyrite nanoparticles; Brassinosteroid (BR) pathway; Amylase activity.

(C) 2020 by the authors. This article is an open-access article distributed under the terms and conditions of the Creative Commons Attribution (CC BY) license (https://creativecommons.org/licenses/by/4.0/).

\section{Funding}

This research received no external funding.

\section{Acknowledgments}

This research has no acknowledgment.

\section{Conflicts of Interest}

The authors declare no conflict of interest. 